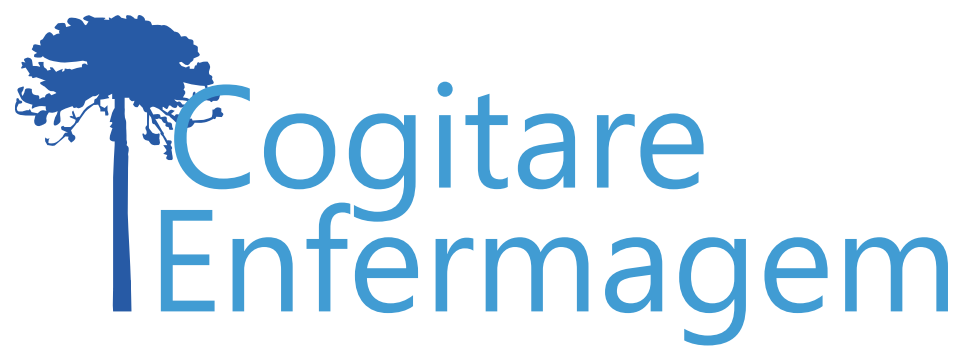

\title{
INTERVENÇÕES NO MANEJO DA FADIGA E QUALIDADE DE VIDA EM PACIENTES EM QUIMIOTERAPIA: ESTUDO DE REVISÃO
}

Lorena Campos Mendes', Elizabeth Barichello²

\section{RESUMO}

Objetivo: identificar na literatura as intervenções não farmacológicas utilizadas no manejo da fadiga oncológica e Qualidade de vida de pacientes com neoplasia digestiva em quimioterapia.

Método: trata-se de uma revisão integrativa. Foram utilizadas as bases de dados MEDLINE, Pubmed, Scopus, CINAHL e Web of Science. Foram selecionados artigos em português, inglês e espanhol entre 2013-2018, que descreviam o uso de intervenções não farmacológicas utilizadas na fadiga oncológica e/ou Qualidade de Vida de pacientes com neoplasia digestiva em quimioterapia. Resultados: do total de 278 artigos, seis foram selecionados. Em relação ao idioma, todos estavam em inglês. Entre os ensaios clínicos, somente um pontuou cinco na Escala de Qualidade de Jadad. Os estudos trouxeram a utilização de programas de atividade física como sendo eficazes.

Conclusão: a prática de atividades física foi considerada uma intervenção eficaz, já a acupuntura não apresentou resposta clínica viável no manejo da fadiga oncológica e Qualidade de Vida.

DESCRITORES: Neoplasias gastrointestinais; Terapias complementares; Fadiga; Qualidade de vida; Enfermagem.

\section{COMO REFERENCIAR ESTE ARTIGO:}

Mendes LC, Barichello E. Intervenções no manejo da fadiga e qualidade de vida em pacientes em quimioterapia: estudo de revisão. Cogitare enferm. [Internet]. 2019 [acesso em "colocar data de acesso, dia, mês abreviado e ano"]; 24. Disponível em: http://dx.doi.org/10.5380/ce.v24i0.61790.

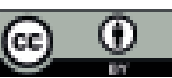

Este obra está licenciado com uma Licença Creative Commons Atribuição 4.0 Internacional. 


\title{
INTERVENTIONS IN THE MANAGEMENT OF FATIGUE AND QUALITY OF LIFE IN PATIENTS UNDERGOING CHEMOTHERAPY: REVIEW STUDY
}

\begin{abstract}
Objective: to identify in the literature the non-pharmacological interventions used in the management of oncological fatigue and Quality of Life of patients with digestive neoplasia undergoing chemotherapy.

Method: this was an integrative review. The MEDLINE, PubMed, Scopus, CINAHL and Web of Science databases were used. Articles in Portuguese, English or Spanish, published between 2013-2018, which described the use of non-pharmacological interventions for oncological fatigue and/or Quality of Life of patients with digestive system neoplasms undergoing chemotherapy were selected.

Results: Of the total of 278 articles retrieved, six were selected. Regarding the language, all were in English. Among the clinical trials, only one scored 5 on the Jadad Quality Scale. The studies indicated the use of physical activity programs as being effective.

Conclusion: the practice of physical activity was considered an effective intervention, while acupuncture did not present a viable clinical response in the management of oncological fatigue and Quality of Life.
\end{abstract}

DESCRIPTORS: Gastrointestinal neoplasms; Complementary therapies; Fatigue; Quality of life; Nursing.

\section{INTERVENCIONES EN EL MANEJO DE LA FATIGA Y CALIDAD DE VIDA EN PACIENTES EN QUIMIOTERAPIA: ESTUDIO DE REVISIÓN}

\section{RESUMEN}

Objetivo: identificar en la literatura las intervenciones no farmacológicas que se utilizan en el manejo de la fatiga oncológica y en la calidad de vida de pacientes con neoplasia digestiva en quimioterapia.

Método: es una revisión integral. Se utilizaron las bases de datos MEDLINE, Pubmed, Scopus, CINAHL y Web of Science. Se eligieron artículos en portugués, inglés y español entre 2013 y 2018, los cuales describían el uso de intervenciones no farmacológicas que se usan para la fatiga oncológica y/o Calidad de Vida de pacientes con neoplasia digestiva en quimioterapia. Resultados: del total de 278 artículos, se seleccionaron seis. Acerca del idioma, todos estaban en inglés. Entre los ensayos clínicos, solamente uno obtuvo cinco puntos en la Escala de Calidad de Jadad. Los estudios mostraron utilización de programas de actividad física como eficaces.

Conclusión: se consideró la práctica de actividades físicas una intervención eficaz, mientas la acupuntura no presentó respuesta clínica viable en el manejo de la fatiga oncológica y en la Calidad de Vida.

DESCRIPTORES: Neoplasias gastrointestinales; Terapias complementarias; Fatiga; Calidad de vida; Enfermería. 
O câncer nas últimas décadas tem ganhado ampla dimensão, convertendo-se em um evidente problema de saúde pública mundial devido a sua relevância epidemiológica, se destacando pela alta taxa de incidência de morbimortalidade. Estimativas para o biênio 2018-2019 apontam a ocorrência de 600 mil casos novos de câncer por ano ${ }^{(1-2)}$.

É preciso considerar a alta incidência e o perfil de morbimortalidade dos tumores do sistema digestório, que se configuram como um dos tipos oncológicos mais prevalentes entre as populações, abrangendo tumores que atingem desde a boca até órgãos como o esôfago, estômago, intestinos delgado e grosso, vesícula biliar, fígado, pâncreas e reto, sendo os mais incidentes a neoplasia colorretal e a neoplasia gástrica ${ }^{(3-5)}$.

Neste contexto, dentre os diferentes tratamentos oncológicos instituídos, a quimioterapia se destaca como uma das modalidades de maior escolha objetivando a cura, controle ou mesmo paliação da doença. Ressalva-se que, por não possuírem especificidade, os quimioterápicos não agridem exclusivamente as células tumorais, podendo causar prejuízos para células normais que possuem características similares às neoplásicas. Em função desta agressão, a quimioterapia antineoplásica desencadeia toxidade ou efeitos colaterais ao organismo, podendo causar efeitos imediatos; precoce; retardado ou tardio ${ }^{(6-7)}$.

Por conseguinte, o tratamento pode ocasionar efeitos colaterais como xerostomia, mucosite, alopecia, inapetência, disfagia, náuseas, vômitos, perda de peso, constipação, fadiga, sonolência, preocupações com a imagem corporal e tristeza. Todos estes sintomas têm um impacto significativo sobre a vida dos pacientes, alterando tanto a sua capacidade de autoestima como a de autocuidado ${ }^{(8)}$.

Destaca-se a fadiga oncológica como um dos sintomas mais prevalentes em pacientes com câncer, sendo referido por $50 \%$ a $90 \%$ dos pacientes, impactando de forma negativa sua Qualidade de Vida (QV) e Capacidade Funcional (CF) diária(9). A fadiga oncológica pode ser definida como um sentimento subjetivo, persistente e angustiante de cansaço cognitivo e/ou emocional, ou ainda exaustão relacionada à doença e/ou o tratamento, interferindo nas atividades cotidianas. Dessa forma, é importante o rastreio da presença de fadiga no paciente oncológico pelos profissionais de saúde desde o diagnóstico até $o$ término do tratamento, bem como da avaliação da $\mathrm{QV}{ }^{(10)}$.

Outro aspecto importante a ser mensurado é a QV do paciente em quimioterapia, uma vez que se destaca como importante recurso para a avaliação dos resultados do tratamento, sendo um instrumento fundamental para o acompanhamento dos pacientes, monitoramento das manifestações clínicas e dos efeitos colaterais e na repercussão da terapêutica com quimioterapia antineoplásica ${ }^{(11)}$.

QV pode ser definida como a percepção dos indivíduos a respeito de sua posição na vida, no contexto cultural e dos sistemas de valores em que vivem e em relação a suas metas, expectativas e preocupações. Além disso, QV é um conceito abrangente afetado de uma maneira complexa pela saúde física, estado psicológico, nível de independência, relações sociais, crenças pessoais e características ambientais ${ }^{(12-13)}$. A mensuração da OV, por conseguinte, tem sido uma importante ferramenta utilizada em ensaios clínicos com quimioterápicos antineoplásicos objetivando avaliar os efeitos colaterais decorrentes do tratamento, destacando-se como um dos melhores métodos para avaliar a tolerância do paciente ao tratamento ${ }^{(14)}$.

Sob este prisma, a busca pela qualidade na assistência aos pacientes oncológicos submetidos ao tratamento com quimioterapia antineoplásica é um aspecto importante a ser mensurado, uma vez que avalia as várias dimensões da doença, além de criar parâmetros clínicos para a prática assistencial(14). Cabe ao profissional de saúde que presta cuidado ao paciente em uso de quimioterápicos fornecer uma assistência adequada à necessidade de cada paciente, de maneira integral e individual, com o intuito de tornar o tratamento menos doloroso para essa população(15). 
Observa-se que pacientes oncológicos em tratamento com quimioterápicos são constantemente submetidos a procedimentos que geram desconforto, surgindo a necessidade da procura por práticas complementares associadas ao tratamento médico tradicional, a fim de minimizar os eventos adversos e aumentar a $\mathrm{QV}{ }^{(16)}$.

Assim, destaca-se a importância da intervenção em saúde a estes pacientes como meio de aconselhamento, objetivando a adaptação e manejo dos sintomas do paciente à fadiga e consequentemente melhorando sua QV. Ressalta-se que uma pessoa capacitada, por meio do empoderamento através de ações educativas, pode melhorar sua compreensão e participar ativamente de seus cuidados, mobilizando recursos e ações que reduzam a ansiedade, fortalecendo suas estratégias para lidar com o câncer e consequentemente melhorar sua $\mathrm{QV}\left({ }^{(17)}\right.$.

Estudo de intervenção demonstrou que a educação sobre os sintomas fornecida a pacientes oncológicos em tratamento quimioterápico diminuiu a frequência, gravidade e grau de desconforto causado pelo tratamento ${ }^{(18)}$.

Analisando a magnitude dos tumores do sistema digestório, a ocorrência da fadiga oncológica e piora da QV em pacientes em tratamento com quimioterapia antineoplásica, considera-se que a contribuição da intervenção em saúde na orientação e na prestação de uma assistência qualificada, pautadas na cientificidade e na consequente otimização do tratamento podem auxiliar na recuperação do paciente oncológico. Portanto o presente estudo teve como objetivo identificar na literatura as intervenções em saúde não farmacológicas utilizadas no manejo da fadiga oncológica e na QV de pacientes com neoplasia do sistema digestório em tratamento quimioterápico.

\section{MÉTODO}

Trata-se de uma pesquisa do tipo revisão integrativa. Para a realização do estudo e alcance do objetivo proposto, foram obedecidas as seguintes etapas: elaboração da pergunta norteadora, estabelecimento dos objetivos e elaboração dos critérios de inclusão e exclusão dos artigos, utilização de instrumento previamente elaborado na coleta das informações dos estudos, seleção dos artigos na literatura, discussão dos resultados e apresentação da revisão integrativa ${ }^{(19)}$.

Para guiar a revisão integrativa, formulou-se a seguinte questão norteadora: "quais são as evidências científicas sobre as intervenções em saúde não farmacológicas utilizadas no manejo da fadiga oncológica e QV em pacientes com neoplasia do sistema digestório em tratamento com quimioterapia antineoplásica".

O levantamento bibliográfico foi realizado no mês de abril de 2018 por meio do acesso às bases de dados eletrônicas MEDLINE, PubMed (Digital Biomedical Archives and Health Sciences of the US National Institutes of Health), Scopus, CINAHL (Cumulative Index to Nursing and Allied Health Literature) e Web of Science. A seleção dos termos da pesquisa foi adaptada para corresponder à ferramenta de pesquisa de cada banco de dados, usando termos MeSH para pesquisar nas bases de dados PubMed, Web of Science, Scopus e CINAHL, e os termos DeCS (Descriptores en Ciencias de la Salud) para MEDLINE. Foram utilizados como descritores os termos "Gastrointestinal Neoplasms"; "Drug Therapy"; "fatigue" e "Clinical Trial". Os termos foram isolados, analisados e combinados com a ajuda do operador booleano "AND".

Foram incluídos artigos em língua portuguesa, inglesa e espanhola, publicados nos anos de 2013 até abril de 2018, em acesso aberto e textos completos, que descreviam o uso de intervenções em saúde não farmacológicas utilizadas no manejo da fadiga oncológica e/ou QV de pacientes com neoplasia do sistema digestório, sendo este exclusivo ou em conjunto com outros tipos de tumores, em tratamento com quimioterapia antineoplásica. Foram excluídos os artigos de revisão e relatos de experiência e os artigos que não 
utilizavam a quimioterapia como tratamento oncológico principal.

No total, 278 (100\%) artigos foram identificados nas cinco bases de dados investigadas sendo: $265(95,3 \%)$ na PubMed, $10(3,6)$ na MEDLINE, dois $(0,7 \%)$ na CINAHL, um $(0,4 \%)$ na Scopus e nenhum (0\%) na Web of Science. Após a busca de dados, seguiu-se com a préseleção dos artigos a partir da leitura de seus títulos e resumos, aqueles que não tinham relação com o assunto, considerando os critérios de inclusão e exclusão, foram descartados. Depois de ler os títulos e resumos, foram selecionados 11 estudos, considerando os critérios de inclusão e exclusão, que foram avaliados para elegibilidade.

A análise do desenho de pesquisa dos estudos incluídos na revisão integrativa foi baseada nos conceitos descritos por Polit e Beck (2016). Os autores classificam os estudos quantitativos em experimental, quase experimentais e não experimental(20). A qualidade metodológica dos ensaios clínicos randomizados foi analisada utilizando a Escala de Qualidade de Jadad, que consiste em uma escala de qualidade para medir o provável viés no estudo. A pontuação de Jadad é aferida por meio de uma escala de cinco pontos incluindo três critérios: randomização, nível de cegamento, e menção de desistências e retiradas. Dos cinco pontos potenciais, são considerados protocolos de alta qualidade aqueles pontuados com escore maior ou igual a três e baixa qualidade menor que três ${ }^{(21)}$.

Os dados foram analisados descritivamente e apresentados em detalhes, incluindo um resumo do conhecimento produzido em cada estudo como objetivo, amostra, intervenção utilizada, principais resultados e conclusões.

Uma tabela de extração de dados foi projetada para agrupar as informações dos estudos, apresentando as seguintes informações: identificação (autores, título, periódico, ano, volume, número, página e idioma), tipo de publicação, delineamento da pesquisa, características da amostra, tratamento dos dados estatísticos, intervenções realizadas, principais resultados e conclusão.

\section{RESULTADOS}

Entre os 11 (100\%) estudos primários selecionados, cinco (45,5\%) foram excluídos por não abordarem o estudo completo e/ou não abordar o tema da pesquisa. Assim, a amostra da revisão consistiu em seis (100\%) estudos primários: cinco (83,3\%) na PubMed e um (16,7\%) na MEDLINE. O processo da análise dos estudos está ilustrado na Figura 1 por meio do fluxograma PRISMA ${ }^{(22)}$. 


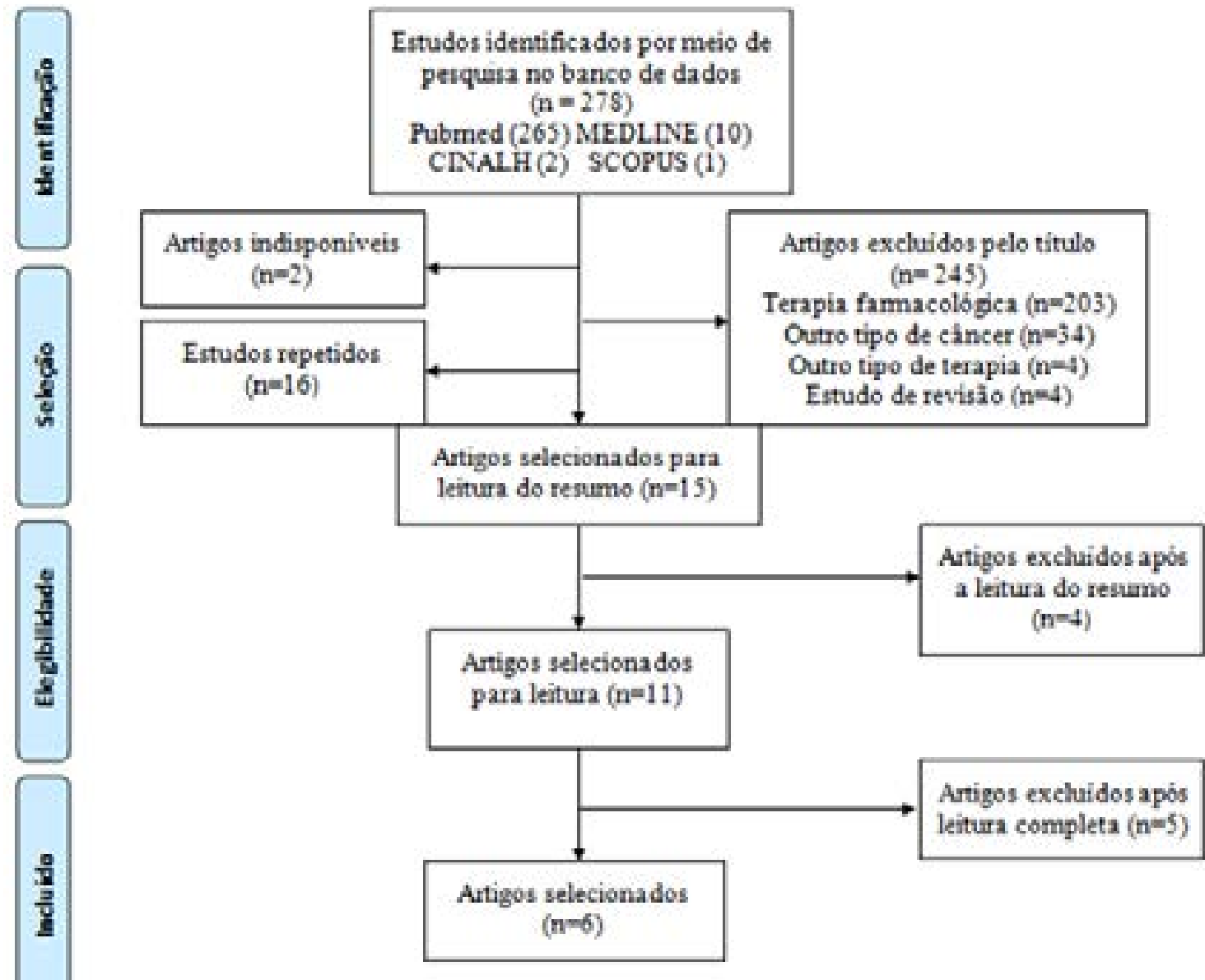

Figura 1 - Fluxo de informações com diferentes fases da revisão integrativa. Adaptado de Moher et al., 2009. Uberaba, MG, Brasil, 2018

Em relação à língua, todos os artigos incluídos estavam em inglês. Quanto ao desenho da pesquisa, cinco $(83,3 \%)$ estudos foram classificados como ensaio clínico randomizado e um $(16,7 \%)$ como quase experimental (Quadro 1).

Quadro 1 - Caracterização dos estudos incluídos na revisão. Uberaba, MG, Brasil, 2018 (continua)

\begin{tabular}{|l|l|l|}
\hline Título & Autores/ano & Tipo de estudo \\
\hline $\begin{array}{l}\text { 1. Feasibility of a 12-month-exercise } \\
\text { intervention during and after radiation and } \\
\text { chemotherapy in cancer patients: impact on } \\
\text { quality of life, peak oxygen consumption, and } \\
\text { body composition }\end{array}$ & $\begin{array}{l}\text { Grabenbauer A, Grabenbauer AJ, } \\
\text { Lengenfelder R, Grabenbauer GG, } \\
\text { Distel LV (2016) }\end{array}$ & $\begin{array}{l}\text { Quase } \\
\text { Experimental }\end{array}$ \\
\hline $\begin{array}{l}\text { 2. Acupuncture for the treatment of post- } \\
\text { chemotherapy chronic fatigue: a randomized, } \\
\text { blinded, sham-controlled trial }\end{array}$ & $\begin{array}{l}\text { Deng Gary, Chan Y, Sjoberg D, } \\
\text { Vickers A, Yeung KS, Kris M, et al. } \\
(2013)^{(24)}\end{array}$ & Ensaio Clínico \\
\hline $\begin{array}{l}\text { 3. Cost-effectiveness analysis of na 18-week } \\
\text { exercise programme for atients with breast } \\
\text { and colon câncer undergoing adjuvant } \\
\text { chemotherapy: the randomised PACT study }\end{array}$ & $\begin{array}{l}\text { May AM, Bosch MJC, Velthuis MJ, } \\
\text { Van der Wall E, Bisschop CNS, Los } \\
\text { M, et al. (2017)(25) }\end{array}$ & Ensaio Clínico \\
Randomizado
\end{tabular}




\begin{tabular}{|l|l|l|}
\hline $\begin{array}{l}\text { 4. A randomized pilot study with daily walking } \\
\text { during adjuvante chemotherapy for patients } \\
\text { with breast and colorectal câncer }\end{array}$ & $\begin{array}{l}\text { Backman Malin, Wengstr Y, } \\
\text { Johansson B, Sköldengen I, } \\
\text { Börjesson S, Tärnbro S, et al. (2014) } \\
\text { (26) }\end{array}$ & $\begin{array}{l}\text { Ensaio Clínico } \\
\text { Randomizado }\end{array}$ \\
\hline $\begin{array}{l}\text { 5. Exercise training in patients with advanced } \\
\text { gastrointestinal cancer undergoing palliative } \\
\text { chemotherapy: a pilot study }\end{array}$ & $\begin{array}{l}\text { Jensen W, Baumann FT, Stein A, } \\
\text { Bloch W, Bokemeyer C, Wit M, et } \\
\text { al. (2014) (27) }\end{array}$ & Ensaio Clínico \\
Randomizado
\end{tabular}

Entre os ensaios clínicos, um estudo (20\%)(24) marcou cinco na Escala de Qualidade de Jadad (pontuação total máxima de cinco pontos), indicando que o estudo foi descrito como randomizado, o método para produzir a randomização estava adequado, descreveu e utilizou um cegamento apropriado e as exclusões foram relatadas. Um estudo $(20 \%)^{(28)}$ recebeu pontuação de três, indicando que o estudo foi descrito como randomizado, o método para produzir a sequência de randomização foi apropriado, e as perdas e exclusões foram relatados, mas sem mascaramento. Dois estudos $(40 \%)^{(26-27)}$ receberam pontuação de dois pontos, uma vez que descreveram a randomização e as retiradas e desistência, e um $(20 \%)^{(25)}$ dos artigos recebeu um ponto, pois descreveu somente a randomização. Os estudos foram publicados entre 2013 e 2017. A análise metodológica segundo Jadad está descrita no Quadro 2.

Quadro 2 - Análise da qualidade metodológica dos ensaios clínicos randomizados segundo Escala de Qualidade de Jadad. Uberaba, MG, Brasil, 2018

\begin{tabular}{|l|c|c|c|c|c|c|}
\hline Estudo & $\begin{array}{c}\text { Descreve } \\
\text { randomização }\end{array}$ & $\begin{array}{c}\text { Randomização } \\
\text { clara }\end{array}$ & $\begin{array}{c}\text { Descreve } \\
\text { cegamento }\end{array}$ & $\begin{array}{c}\text { Cegamento } \\
\text { adequado }\end{array}$ & $\begin{array}{c}\text { Descreve } \\
\text { retirada e } \\
\text { desistências }\end{array}$ & Jadad \\
\hline Deng et al (2013) & Sim & Sim & Sim & Sim & Sim & 5 \\
\hline May et al (2017) & Sim & Não & Não & Não & Não & 1 \\
\hline Backman et al (2014) & Sim & Não & Não & Não & Sim & 2 \\
\hline Jensen et al (2014) & Sim & Não & Não & Não & Sim & 2 \\
\hline Vulpen et al (2016) & Sim & Sim & Não & Não & Sim & 3 \\
\hline
\end{tabular}

Em relação às intervenções utilizadas no manejo da fadiga e $\mathrm{QV}$, a mais citada foi a utilização de exercícios físicos. Neste contexto, um estudo objetivou avaliar a viabilidade do impacto de exercícios físicos em uma população de pacientes oncológicos durante e após o tratamento com quimioterapia e radioterapia. A amostra foi composta por 45 pacientes, maiores de 18 anos e com motivação para a prática de exercícios físicos. A intervenção realizada consistiu na realização de três sessões semanais de exercícios individuais com duração de 30 a 60 min com aumento gradual da intensidade. Os participantes foram encorajados a continuar o programa de exercícios em casa e a adesão foi monitorada por meio de diários de exercícios, visitas de acompanhamento e/ou telefonemas. Os resultados da pesquisa evidenciaram um aumento nos escores globais do estado de saúde, bem como melhora do consumo de oxigênio, composição corporal e $\mathrm{QV}^{(23)}$. 
Outro ensaio clínico realizado objetivou avaliar o custo-efetividade de um programa de 18 semanas de atividade física durante o tratamento para pacientes com câncer de mama e cólon. Para isso, foram selecionados 237 pacientes randomizados em dois grupos. Para o grupo de intervenção foi oferecido um programa de exercícios de 18 semanas, individualizado para as preferências pessoais e nível de condicionamento físico. As aulas de exercício de uma hora incluíam aquecimento ( $5 \mathrm{~min}$ ), treinamento de força aeróbica e muscular (50 $\mathrm{min})$ e resfriamento $(5 \mathrm{~min})$. Além do programa de exercícios supervisionado, os pacientes foram orientados a manterem-se ativos por $30 \mathrm{~min}$ em pelo menos três dias na semana. Ao finalizar o estudo, os autores concluíram que houve melhora da fadiga nos pacientes com câncer de cólon, além de redução dos custos de quimioterapia, hospitalização, contatos médicos e ausências do trabalho( ${ }^{(25)}$.

Estudo semelhante investigou o efeito de um programa de exercícios supervisionados por 18 semanas durante a quimioterapia adjuvante na fadiga em pacientes com câncer de cólon. $O$ estudo foi realizado com 33 pacientes. Os pacientes randomizados para o grupo de intervenção participaram de um programa de exercícios, sendo supervisionados duas vezes por semana, com duração de uma hora, que incluiu aquecimento (10 min), treinamento de força muscular e atividade aeróbica $(40 \mathrm{~min}$ ) e resfriamento $(10 \mathrm{~min})$. Além das sessões supervisionadas, os pacientes foram instruídos a estar fisicamente ativos por pelo menos 30 minutos, em três outros dias da semana. O estudo mostrou resultados promissores, indicando que um programa de exercícios é viável, seguro, e pode ter efeitos benéficos na fadiga física e fadiga geral|(28).

Outro estudo estudou a viabilidade e aderência da atividade física em pacientes com câncer durante a quimioterapia. Para isso foram selecionados 77 pacientes, randomizados em dois grupos. Os participantes foram incentivados a andar 10000 passos por dia, o que equivale a aproximadamente $8 \mathrm{~km}$ de caminhada diária, por dez semanas. Como resultado, o estudo não encontrou diferenças significativas na QV entre o grupo de intervenção e o controle e reforça como medidas para melhorar os resultados um programa com maior intensidade de atividade física, exercícios supervisionados, incluindo programas individualizados; a inclusão de medidas mais objetivas para avaliar o desempenho; e amostras maiores para aumentar o valor das intervenções de atividade física ${ }^{(26)}$.

Estudo piloto com 21 pacientes buscou investigar a viabilidade de dois programas de treinamento diferentes em pacientes com câncer gastrointestinal submetido à quimioterapia paliativa. Os pacientes foram aleatoriamente alocados em dois grupos: um grupo de resistência e outro de treinamento aeróbico. O grupo de resistência recebeu sessões de treinamento por 45 min supervisionados, incluindo períodos de aquecimento e descanso, duas vezes por semana até um total de 24 sessões durante um período de 12 semanas. Já o grupo de treinamento aeróbico recebeu sessões de $45 \mathrm{~min}$ em bicicleta ergométrica duas vezes por semana durante 12 semanas. Como resultados, o estudo encontrou que o grupo de treinamento aeróbico apresentou melhorias nos escores de QV para o estado de saúde global e função funcional. Já o escore de fadiga diminuiu e a qualidade do sono aumentou em ambos os grupos ${ }^{(27)}$.

Outra intervenção analisada foi a acupuntura, apresentada em apenas um artigo, com o objetivo de determinar a redução da fadiga pós-quimioterapia. Foram selecionados 101 pacientes, divididos em dois grupos: intervenção e controle. Os pacientes de ambos os grupos receberam tratamento uma vez por semana durante seis semanas. No grupo de intervenção foram utilizadas 14 agulhas filiformes inseridas em profundidade e pontos predefinidos. No grupo controle, foram utilizadas agulhas simuladas com ponta romba e os pontos de inserção distante dos pontos usados na acupuntura verdadeira. Os autores concluíram que a acupuntura, tal como utilizada no estudo, não é uma opção clínica viável no tratamento da fadiga crônica pós-quimioterapia. Porém ressaltam como limitações o número de pacientes perdidos durante o acompanhamento e não excluem a possibilidade de um regime de tratamento eficaz utilizando acupuntura(24). 
O estudo e a avaliação da QV e fadiga de pacientes com câncer vêm recentemente despertando o interesse de pesquisadores, uma vez que o câncer se destaca como um problema de saúde pública de proporção mundial, cada vez mais comum entre as populações.

A quimioterapia antineoplásica, devido a sua inespecificidade, ocasiona efeitos colaterais ao paciente em tratamento. De acordo com o Instituto Nacional do Câncer (INCA), alguns desses efeitos são tão prejudiciais ao organismo humano que podem indicar a interrupção do tratamento ou ainda acarretar a morte do paciente, assim, esses possíveis efeitos devem ser previstos, detectados e tratados o mais precocemente possíve ${ }^{(7)}$.

Estudo realizado com pacientes em tratamento quimioterápico contra o câncer observou que a terapêutica instituída desencadeou efeitos colaterais como dor, fadiga, insônia e perda de apetite, causando déficits nas funções desempenhadas e prejudicando a QV dos pacientes em tratamento. Os autores ressaltam que a melhoria na QV desses pacientes pode ocorrer na medida em que os sintomas do tratamento possam ser evitados e controlados, além da adesão a medidas complementares eficazes que possam auxiliar os pacientes a lidarem melhor com a doença e a terapêutica recebida(29).

Corroborando com esses dados, o INCA destaca a importância da avaliação precoce da fadiga, por meio de critérios clínicos de intensidade e limitações biopsicossociais, mensurada por meio de escalas validadas e tratada não só por sua ocorrência, mas também pela maneira como afeta o status funcional do paciente, podendo inclusive interferir em sua decisão quanto ao tratamento proposto $^{(7)}$.

Neste contexto, esta pesquisa avaliou quais as evidências científicas sobre as intervenções em saúde não farmacológicas utilizadas no manejo da fadiga oncológica e QV em pacientes com neoplasia do sistema digestório em tratamento com quimioterapia antineoplásica. Do total de 278 artigos encontrados, seis preencheram os critérios de elegibilidade. Três (50\%) $)^{(23,25,28)}$ sugeriram que a intervenção realizada foi eficaz na melhora da QV e/ou fadiga, dois $(33 \%)^{(24,26)}$ não encontraram melhora nos escores de QV e/ou fadiga, não sugerindo o tratamento como uma opção clínica viável, e um (17\%)(27) que avaliou dois tipos de intervenção diferentes identificou que ambas foram efetivas.

Os estudos incluídos na revisão trazem como terapias utilizadas no manejo da fadiga oncológica e QV a utilização de programas de atividade física de diferentes modos e intensidade como sendo eficazes. Do total de cinco artigos que avaliaram o uso da atividade física, quatro (80\%) consideraram a prática de exercícios físicos como sendo viável para o paciente oncológico em tratamento com quimioterapia antineoplásica, melhorando os escores de QV e/ou fadiga, e trazendo outros benefícios como melhora do consumo de oxigênio, composição corporal, redução dos custos com quimioterapia, hospitalização, contatos médicos, ausência do trabalho e qualidade do sono(23,25,27-28).

Corroborando com os dados encontrados, um estudo traz que, embora uma pequena parcela de pacientes receba orientação da implementação de uma rotina de atividade física, a realização de exercícios físicos se destaca como uma das medidas mais eficazes auxiliando na melhora da fadiga, além de trazer benefícios para a QV, CF e estresse ${ }^{(9)}$.

Já a utilização da acupuntura, exposta por somente um artigo (16\%), não apresentou uma resposta clínica viável em relação a melhoras significativas no escore de fadiga do paciente oncológico. Porém os autores trazem como limitação do estudo o número de pacientes perdidos no seguimento, e não excluem a possibilidade de que um regime de tratamento mais intensivo possa ser eficaz ${ }^{(24)}$.

Outro estudo que avaliou e selecionou diretrizes sobre o tema corrobora com os dados encontrados e ressalta a importância da prática de atividade física, e, diferente do resultado dessa pesquisa, orienta a utilização da acupuntura como estratégia de gerenciamento da fadiga. Além disso, reforça como medidas utilizadas no manejo da fadiga a educação, aconselhamento e orientação sobre possíveis estratégias, o uso da Terapia Cognitivo Comportamental (TCC) e da psicoeducação, além de atividades como o 
Yoga, musicoterapia, Reiki e massagem ${ }^{(10)}$.

Além das intervenções citadas (atividade física e acupuntura), outros tipos de terapêuticas não farmacológicas são utilizadas no manejo da fadiga e listadas na literatura, como a utilização da Terapia Cognitivo Comportamental, terapias de higiene do sono e o uso da medicina alternativa complementar ${ }^{(9)}$. Outro estudo traz ainda a utilização da visualização com imagem guiada e a prática de oração como sendo efetivas na diminuição dos efeitos da quimioterapia ${ }^{(30)}$.

Ressalta-se a importância desses dados, uma vez que essas medidas podem ser efetivas e auxiliar no planejamento da assistência, contribuído para a prática clínica e consequentemente melhorar a $\mathrm{QV}$ e reduzir a fadiga do paciente oncológico em tratamento quimioterápico.

Ressalta-se como limitação do estudo o período de tempo avaliado de cinco anos. Embora tenham sido encontrados 278 artigos, somente seis compuseram a revisão por se adequarem aos critérios de inclusão e exclusão, podendo assim ter sido excluídos artigos publicados anteriormente.

\section{CONCLUSÃO}

É escasso o número de publicações em periódicos científicos a respeito de intervenções não farmacológicas para os pacientes em tratamento quimioterápico com câncer gastrointestinal. Dentre os estudos encontrados, a prática de atividades física foi considerada uma intervenção viável no manejo da fadiga oncológica e melhora na OV de pacientes em tratamento com quimioterapia antineoplásica para neoplasias do sistema digestório.

Ressalta-se que a quimioterapia antineoplásica ocasiona efeitos colaterais ao paciente em tratamento que são prejudiciais ao organismo humano, indicando a interrupção do tratamento ou podendo acarretar a morte do paciente. Este cenário sugere a necessidade de pesquisas sobre esse impacto e a análise de medidas que podem ser utilizadas no manejo desses sintomas.

Desta forma, o desenvolvimento deste trabalho contribuiu na análise dessas medidas objetivando atender a necessidade da assistência e contribuir na adequação do perfil profissional da área da saúde, no que tange ao desenvolvimento de habilidades e competências para atender as necessidades destes clientes.

\section{REFERÊNCIAS}

1. Ministério da Saúde. Instituto Nacional de Câncer. Estimativa 2014: incidência de câncer no Brasil. [Internet]. Rio de Janeiro: Ministério da Saúde; 2014. [acesso em 14 abr 2018]. Disponível em: http://www. saude.sp.gov.br/resources/ses/perfil/gestor/homepage/outros-destaques/estimativa-de-incidencia-decancer-2014/estimativa cancer 24042014.pdf.

2. Ministério da Saúde. Instituto Nacional de Câncer. Estimativa 2018: incidência de câncer no Brasil. [Internet]. Rio de Janeiro: Ministério da Saúde; 2018 [acesso em 14 abr 2018]. Disponível em: http://www. inca.gov.br/estimativa/2018/estimativa-2018.pdf.

3. Araújo ES, Duval PA, Silveira, DH. Sintomas relacionados à diminuição de ingestão alimentar em pacientes com neoplasia do aparelho digestório atendidos por um programa de internação domiciliar.

Rev. bras. cancerol. [Internet]. 2012 [acesso em 18 jun 2018]; 58(4). Disponível em: http://www1.inca.gov. $\mathrm{br} / \mathrm{rbc} / \mathrm{n}$ 58/v04/pdf/09-artigo-sintomas-relacionados-diminuicao-ingestao-alimentar-pacientes-neoplasiaaparelho-digestorio-atendidos-programa-internacao-domiciliar.pdf. 
4. Almeida, GAS. Distúrbios no padrão do sono e fadiga em pacientes submetidos à cirurgia oncológica gastrointestinal [dissertação]. Uberaba (MG): Universidade Federal do Triângulo Mineiro; 2013. Disponível em: http://bdtd.uftm.edu.br/handle/tede/120.

5. Vieira AR, Fortes RC. Qualidade de vida de pacientes com câncer gastrointestinal. Com. Ciências Saúde. [Internet]. 2015 [acesso em 14 abr 2018]; 26(1/2). Disponível em: http://bvsms.saude.gov.br/bvs/ periodicos/ccs artigos/2015 qualidade vida_pacientes.pdf.

6. Silva SR, Aguillar OM. Assistência de enfermagem em quimioterapia antineoplásica. Petrópolis: EPUB; 2001.

7. Ministério da Saúde. Instituto Nacional de Câncer. Ações de enfermagem para o controle do câncer: uma proposta de integração ensino-serviço. [online]. Rio de Janeiro: Ministério da Saúde; 2008 [acesso em 16 abr 2018]. Disponível em: http://bvsms.saude.gov.br/bvs/publicacoes/acoes enfermagem controle cancer.pdf.

8. Silva JMC, Pontifice PS. Estrategias para el autocuidado de las personas con cáncer que reciben quimioterapia / radioterapia y su relación con el bienestar. Enferm. glob. [Internet]. 2015 [acesso em 16 abr 2018]; 14(37). Disponível em: http://scielo.isciii.es/pdf/eg/v14n37/revision2.pdf.

9. Campos MP de O, Hassan BJ, Riechelmann R, Del Giglio A. Cancer-related fatigue: a review. Rev. Assoc. Med. Bras. [Internet]. 2011 [acesso em 19 abr 2018]; 57(2). Disponível em: http://www.scielo.br/ $\mathrm{pdf} / \mathrm{ramb} / \mathrm{v} 57 \mathrm{n} 2 / \mathrm{en}$ v57n2a21.pdf.

10. Bower JE, Bak K, Berger A, Breitbart W, Escalante CP, Ganz PA, et al. Screening, Assessment, and Management of Fatigue in Adult Survivors of Cancer: An American Society of Clinical Oncology Clinical Practice Guideline Adaptation. J. Clin. Oncol. [Internet]. 2014 [acesso em 04 jul 2018]; 32(17). Disponível em: http://ascopubs.org/doi/pdf/10.1200/JCO.2013.53.4495.

11. Chaves PL, Gorini MIPC. Qualidade de vida do paciente com câncer colorretal em quimioterapia ambulatorial. Rev. Gaúch. Enferm. [Internet]. 2011 [acesso em 11 maio 2018]; 32(4). Disponível em: http:// www.scielo.br/pdf/rgenf/v32n4/v32n4a18.pdf.

12. The Whoqol Group. The World Health Organization Quality of Life Assessment (whoqol): position paper from The world health organization. Soc Sci Med. [Internet]. 1995 [acesso em 19 abr 2018]; 41(10). Disponível em: https://www.ncbi.nlm.nih.gov/pubmed/8560308.

13. World Health Organization (WHO). Whoqol: measuring quality of life. [Internet]. 1997 [acesso em 01 jun 2018]. Disponível em: https://www.who.int/healthinfo/survey/whoqol-qualityoflife/en/.

14. Terra F de S, Costa AMDD, Damasceno L de L, Lima T da SL, Filipini CB, Leite MAC. Avaliação da qualidade de vida de pacientes oncológicos submetidos à quimioterapia. Rev Bras Clin Med. [Internet]. 2013 [acesso em 01 jun 2018]; 11(2). Disponível em: http://files.bvs.br/upload/S/1679-1010/2013/v11n2/ a3564.pdf.

15. Elias TC, Mendes LC, Soares MBO, Haas VJ, Silva SR da. Influência de variáveis clínicas na capacidade funcional de mulheres em tratamento quimioterápico. Esc. Anna Nery [Internet]. 2015 [acesso em 01 jun 2018]; 19(4). Disponível em: http://dx.doi.org/10.5935/1414-8145.20150076.

16. Jaconodino CB, Amestoy SC, Thofehrn MB. A utilização de terapias alternativas por pacientes em tratamento quimioterápico. Cogitare enferm. [Internet]. 2008 [acesso em 01 jun 2018]; 13(1). Disponível em: http://dx.doi.org/10.5380/ce.v13i1.11953.

17. Maunsell E, Lauzier S, Brunet J, Pelletier S, Osborne RH, Campbell HS. Health-Related Empowerment in Cancer: Validity of Scales from the Health Education Impact Questionnaire. Cancer. [Internet]. 2014 [acesso em 14 abr 2018]; 120(2). Disponível em: https://doi.org/10.1002/cncr.28847.

18. Sahin Z, Ergüney S. Effect on Symptom Management Education Receiving Patients of Chemotherapy. J Cancer Educ [Internet]. 2016 [acesso em 04 jul 2018]; 31. Disponível em: https://link.springer.com/ article/10.1007\%2Fs13187-015-0801-8. 
19. Souza MT de, Silva MD da, Carvalho R de. Revisão integrativa: o que é e como fazer. Einstein. [Internet]. 2010 [acesso em 14 abr 2018]; 8(1). Disponível em: http://www.scielo.br/pdf/eins/v8n1/ pt 1679-4508-eins-8-1-0102.pdf.

20. Polit DF, Beck CT. Fundamentos de pesquisa em enfermagem: avaliação de evidências para a prática da enfermagem. 7. ed. Porto Alegre: Artmed; 2011.

21. Jadad AR, Moore RA, Carrol D, Jenkinson C, Reynolds DJ, Gavaghan DJ, et al. Assessing the quality of reports of randomized clinical trials: is blinding necessary? Control Clin Trials. [Internet]. 1996 [acesso em 08 jul 2018]; 17(1). Disponível em: https://www.ncbi.nlm.nih.gov/pubmed/8721797.

22. Moher D, Liberati A, Tetzlaff, J, Altman DG. Preferred reporting items for systematic reviews and meta-analyses: The PRISMA statement. PloS Med. [Internet]. 2009 [acesso em 14 abr 2018]; 6(7).

Disponível em: https://doi.org/10.1371/journal.pmed.1000097.

23. Grabenbauer A, Grabenbauer AJ, Lengenfelder R, Grabenbauer GG, Distel LV. Feasibility of a 12-month-exercise intervention during and after radiation and chemotherapy in cancer patients: impact on quality of life, peak oxygen consumption, and body composition. Radiat. oncol. [Internet]. 2016 [acesso em 16 abr 2018]; 11(42). Disponível em: https://doi.org/10.1186/s13014-016-0619-5.

24. Deng Gary, Chan Y, Sjoberg D, Vickers A, Yeung KS, Kris M, et al. Acupuncture for the treatment of post chemotherapy chronic fatigue: a randomized, blinded, sham-controlled trial. Support Care Cancer [Internet]. 2013 [acesso em 14 abr 2018]; 21(6). Disponível em: https://doi.org/10.1007/s00520-0131720-z.

25. May AM, Bosch MJC, Velthuis MJ, Wall E Van der, Bisschop CNS, Los M, et al. Cost-effectiveness analysis of an 18-week exercise programme for patients with breast and colon cancer undergoing adjuvant chemotherapy: the randomised PACT study. BMJ Open. [Internet]. 2017 [acesso em $16 \mathrm{abr}$ 2018]; 7. Disponível em: https://doi.org/10.1136/bmjopen-2016-012187.

26. Backman M, Wengstrom Y, Johansson B, Sköldengen I, Börjesson S, Tärnbro S, et al. A randomized pilot study with daily walking during adjuvante chemotherapy for patients with breast and colorectal câncer. Acta Oncologica. [Internet]. 2014 [acesso em 21 abr 2018]; 53(4). Disponível em: https://doi. org/10.3109/0284186X.2013.873820.

27. Jensen W, Baumann FT, Stein A, Bloch W, Bokemeyer C, Wit M, et al. Exercise training in patients with advanced gastrointestinal cancer undergoing palliative chemotherapy: a pilot study. Support Care Cancer. [Internet]. 2014 [acesso em 08 jul 2018]; 22(7). Disponível em: https://link.springer.com/ article/10.1007\%2Fs00520-014-2139-x.

28. Vulpen JKV, Velthuis MJ, Bisschop CNS, Travier N, Buijs BJWVD, Backx FJG, et al. Effects of an Exercise Program in Colon Cancer Patients undergoing Chemotherapy. Med Sci Sports Exerc [Internet]. 2016 [acesso em 05 jul 2018]; 48(5). Disponível em: https://doi.org/10.1249/MSS.0000000000000855.

29. Nicolussi AC, Sawada NO, Cadozo FMC, Andrade V, Paula JM. Qualidade de vida relacionada à saúde de pacientes com câncer em quimioterapia. Rev RENE. [Internet]. 2014 [acesso em 04 jul 2018]; 15(1). Disponível em: https://doi.org/10.15253/2175-6783.2014000100017.

30. Sawada NO, Nicolussi AC, Paula JM de, Garcia-Caro MP, Marti-Garcia C, Cruz-Quintana F. Qualidade de vida de pacientes brasileiros e espanhóis com câncer em tratamento quimioterápico: revisão integrativa da literatura. Rev. Latino-Am. Enfermagem. [Internet]. 2016 [acesso em 04 jul 2018]; 24. Disponível em: https://doi.org/10.1590/1518-8345.0564.2688. 
Autor Correspondente:

Elizabeth Barichello

Universidade Federal do Triângulo Mineiro

Pç. Manoel Terra, 330 - 38015050 - Uberaba, MG, Brasil

E-mail: lizabarichello@hotmail.com

\section{Contribuição dos autores:}

Contribuições substanciais para a concepção ou desenho do estudo; ou a aquisição, análise ou interpretação de dados do estudo - LCM, EB

Elaboração e revisão crítica do conteúdo intelectual do estudo - LCM

Aprovação da versão final do estudo a ser publicado - LCM, EB

Responsável por todos os aspectos do estudo, assegurando as questões de precisão ou integridade de qualquer parte do estudo - LCM, EB 